\title{
Tuberculose peritoneal: experiência em um hospital geral
}

\author{
Tuberculous peritonitis: experience in a community hospital
}

Leonardo Gomes da Fonseca ${ }^{a}$, Fernando Peixoto Ferraz de Campos ${ }^{b}$, Aloísio Felipe-Silva

Fonseca LG, Campos FPF, Felipe-Silva A. Tuberculose peritoneal: experiência em um hospital geral. Autopsy Case Rep [Internet]. 2011;1(2):3-9.

\section{RESUMO}

\begin{abstract}
A tuberculose peritoneal apresenta altas taxas de morbimortalidade e o prognóstico dos pacientes depende do diagnóstico precoce. Os sintomas mimetizam outras doenças abdominais, o que torna o diagnóstico difícil. Revisamos dados de 10 pacientes internados no Hospital Universitário da Universidade de São Paulo entre 2003 e $2010 \mathrm{com}$ diagnóstico de tuberculose peritoneal. Dor abdominal, febre e perda de peso foram os sintomas mais frequentes e ascite foi observada em metade dos casos. A dosagem do gradiente soro-ascítico de albumina e a atividade da adenosina deaminase (ADA) auxiliaram o diagnóstico. A cultura de bacilo álcool-ácido resistente no líquido ascítico revelou baixa sensibilidade. $O$ achado de serosite crônica granulomatosa com necrose caseosa e a pesquisa do bacilo álcool-ácido resistente nas biópsias obtidas através de laparotomia ou laparoscopia foram determinantes para o diagnóstico.
\end{abstract}

Unitermos: Tuberculose; Peritonite; Adenosina desaminase; Serosite.

\begin{abstract}
Peritoneal tuberculosis presents high morbi-mortality rates and its prognosis depends on early diagnosis. Symptoms mimic other abdominal diseases, what makes diagnosis difficult. We report clinical and pathological data from 10 tuberculous peritonitis patients admitted to Hospital Hospital Universitário da Universidade de São Paulo between 2003 and 2010. Abdominal pain, fever and weight loss were the most frequent symptoms and ascites was observed in half of cases. The serum-ascites albumin gradient determination and adenosine deaminase activity helped to make the diagnosis. Culture growth of Mycobacterium tuberculosis showed low sensitivity. The finding of chronic granulomatous serositis and positive stain for acid fast bacilli in biopsy specimens obtained from laparotomy and laparoscopy were determinant for diagnosis.
\end{abstract}

Keywords: Tuberculosis; Peritonitis; Adenosine deaminase; Serositis.

\footnotetext{
a Hospital das Clínicas da Faculdade de Medicina - Universidade de São Paulo, São Paulo/SP, Brasil.

b Divisão de Clínica Médica do Hospital Universitário - Universidade de São Paulo, São Paulo/SP, Brasil.

c Serviço de Anatomia Patológica do Hospital Universitário - Universidade de São Paulo, São Paulo/SP, Brasil.
} 


\section{INTRODUÇÃO}

A tuberculose peritoneal é uma forma rara de tuberculose extrapulmonar descrita pela primeira vez em 1843. ${ }^{1}$ Dentre o total de casos diagnosticados de tuberculose, o comprometimento peritoneal representa 0,1 a $0,7 \%$ de todos os casos, sendo mais comum entre os 35 e 45 anos de idade. Dados americanos mostram que o peritônio é o sexto sítio mais acometido pela tuberculose e, em 3,5\% dos casos de doença pulmonar, há concomitância com doença peritoneal. $^{2}$

A incidência das formas extrapulmonares de tuberculose cresce mesmo em países desenvolvidos, principalmente devido a coinfecção com o vírus HIV. Nos Estados Unidos, houve crescimento de $20 \%$ num período de 5 anos. ${ }^{3}$ Algumas condições aumentam o risco do comprometimento peritoneal pela tuberculose como: diálise peritoneal, hepatopatias, carcinomatose peritoneal, neoplasia ovariana e ascite por hipertensão portal. ${ }^{4}$

A tuberculose peritoneal se desenvolve mais comumente a partir da reativação de focos latentes no peritônio, e mais raramente por disseminação transmural ou contiguidade com a cavidade peritoneal. Pode ser dividida em três tipos: (1) "úmida", com ascite livre ou loculada; (2) "seca", com nódulos caseosos e fibrose peritoneal; e (3) fibrótica, com agrupamento de alças, massas omentais e aderências. A tuberculose peritoneal é a forma mais comum de tuberculose abdominal. ${ }^{4,5}$

O diagnóstico da doença é um desafio, dado que as características clínicas costumam ser inespecíficas e mimetizam outras doenças abdominais. Além disso, o isolamento da micobactéria no liquido ascítico é difícil, sendo muitas vezes necessário o auxilio da laparoscopia para o diagnóstico. Chu et al., por meio de biópsia do peritônio guiada por laparoscopia, identificaram a tuberculose peritoneal como causa de $20 \%$ dos casos de ascite de origem obscura. ${ }^{6}$

Em série de casos com pacientes em diálise peritoneal, o intervalo entre a apresentação clínica e o diagnóstico foi de 6,8 semanas. O atraso no diagnóstico e, consequentemente, no início da terapêutica, está relacionado com pior prognóstico de pacientes com tuberculose peritoneal. ${ }^{7}$

Apresentamos uma série de 10 casos de tuberculose peritoneal internados no Hospital Uni- versitário da Universidade de São Paulo (HU-USP), destacando a apresentação clínica, os fatores de risco associados e a forma de diagnóstico.

\section{Casuística e Métodos}

Foram analisados prontuários de pacientes internados no HU-USP entre o período de janeiro de 2003 a dezembro de 2010 que receberam alta com diagnóstico de tuberculose peritoneal. ${ }^{d}$ Os seguintes dados foram coletados: quadro clínico e duração dos sintomas, antecedentes mórbidos, dados de exame físico, análise bioquímica e microbiológica de líquido ascítico (quando presente), e análise histopatológica das amostras de tecidos obtidas por meio de laparoscopia ou laparotomia. As amostras foram coradas com hematoxilinaeosina e Ziehl-Neelsen para pesquisa de bacilo álcool-ácido resistente.

\section{RESULTADOS}

Dos 10 pacientes com diagnóstico de tuberculose peritoneal, 8 (80\%) eram do sexo masculino e $2(20 \%)$ feminino. A idade variou de 18 a 59 anos com média de 36,3 anos e mediana de 35 anos. 0 tempo de duração variou de 3 dias a 7 meses.

Em ordem decrescente, os sintomas mais comuns foram: dor abdominal $(80 \%)$, febre $(60 \%)$, perda de peso (50\%) e diarréia (30\%). Ao exame físico, ascite foi o sinal mais comum, presente em $50 \%$. Etilismo esteve presente em 4 (40\%), e tuberculose em outro sítio em 2 (20\%) pacientes, um com tuberculose pulmonar diagnosticada e tratada previamente ao diagnóstico de tuberculose peritoneal (Caso 3) e outro com tuberculose adrenal concomitante (Caso 5), nenhum paciente apresentou sorologia positiva para HIV. Em 2 casos que relatamos, a apresentação inicial foi de abdômen agudo, um perfurativo (Caso 10) e outro obstrutivo (Caso 6).

A análise do líquido ascítico, que foi realizada em $5(50 \%)$ pacientes, mostrou celularidade variando entre 363 e 3080 células $/ \mathrm{mm}^{3}$ e em todos os casos houve predomínio linfocítico. A dosagem da adenosina deaminase (ADA) variou entre 29 e 113U/l e o gradiente soro-ascite de albumina foi superior a 1,1 em 2 casos (Casos 1 e 2) e inferior em 3 casos (Ca$\operatorname{sos} 4,5$ e 8$)$.

${ }^{\mathrm{d}}$ Dados fornecidos pelo Serviço de Informática do HU-USP 
Oitenta por cento dos pacientes foram submetidos à laparotomia exploradora e $20 \%$ à laparoscopia para obtenção de amostras para biópsia, com pesquisa de bacilo álcool-ácido resistente positiva em $70 \%$ dos casos. Todos os pacientes receberam tratamento específico de acordo com as recomendações do Ministério da Saúde para o tratamento de tuberculose. Somente um paciente apresentou hepatotoxicidade pelo tratamento específico, paciente este que era dependente de drogas ilícitas e que veio a falecer durante a internação por quadro séptico e sangramento digestivo. Noventa por cento dos pacientes receberam alta hospitalar para seguimento em Unidade Básica de Saúde. As tabelas 1 e 2 resumem os dados obtidos na pesquisa.

Tabela 1 - Características clínicas e laboratoriais dos pacientes

\begin{tabular}{|c|c|c|c|c|c|c|c|c|c|c|}
\hline & Caso 1 & Caso 2 & Caso 3 & Caso 4 & Caso 5 & Caso 6 & Caso 7 & Caso 8 & Caso 9 & Caso 10 \\
\hline Sexo & M & $\mathrm{F}$ & $\mathrm{F}$ & M & $\mathrm{M}$ & M & M & M & M & M \\
\hline $\begin{array}{l}\text { Idade } \\
\text { (anos) }\end{array}$ & 31 & 28 & 43 & 36 & 34 & 48 & 18 & 28 & 59 & 38 \\
\hline $\begin{array}{l}\text { Duração } \\
\text { dos sin- } \\
\text { tomas }\end{array}$ & 2 meses & 4 semanas & $\begin{array}{l}4 \text { sema- } \\
\text { nas }\end{array}$ & 1 semana & 3 meses & 5 dias & 15 dias & 15 dias & 7 meses & 3 dias \\
\hline Febre & - & + & - & + & + & - & + & + & + & - \\
\hline $\begin{array}{l}\text { Perda de } \\
\text { peso }\end{array}$ & - & + & - & - & + & - & + & + & - & - \\
\hline Diarréia & - & - & - & - & + & - & - & + & - & - \\
\hline $\begin{array}{l}\text { Dor ab- } \\
\text { dominal }\end{array}$ & + & + & + & + & - & + & + & - & + & + \\
\hline Ascite & + & + & - & + & + & - & - & + & - & - \\
\hline $\begin{array}{l}\text { Tubercu- } \\
\text { lose em } \\
\text { outro sítio }\end{array}$ & - & - & + & - & + & - & - & - & - & - \\
\hline Etilismo & + & - & - & + & + & - & - & - & + & - \\
\hline HIV & - & - & - & - & - & - & - & - & - & - \\
\hline \multicolumn{11}{|l|}{$\begin{array}{l}\text { Líquido } \\
\text { ascitico }\end{array}$} \\
\hline ADA (U/L) & 113 & 75,5 & & 75,9 & 99 & & & 29 & & \\
\hline $\begin{array}{l}\text { GASA }(g / \\
\mathrm{dL})\end{array}$ & 1,3 & 1,6 & & 0,8 & 0,1 & & & 0,3 & & \\
\hline (linfócitos) & $\begin{array}{l}693 \\
(367)\end{array}$ & 1550(992) & & $363(283)$ & $3080(1755)$ & & & $\begin{array}{l}1440 \\
(1022)\end{array}$ & & \\
\hline $\begin{array}{l}\text { pBAAR/ } \\
\text { cBAAR }\end{array}$ & $-/+$ & $-/-$ & & $-/-$ & NR & & & $-/-$ & & \\
\hline \multicolumn{11}{|l|}{ Biópsia } \\
\hline $\begin{array}{l}\text { Método de } \\
\text { coleta }\end{array}$ & $\begin{array}{l}\text { laparoto- } \\
\text { mia }\end{array}$ & $\begin{array}{l}\text { laparosco- } \\
\text { pia }\end{array}$ & $\begin{array}{l}\text { laparo- } \\
\text { tomia }\end{array}$ & $\begin{array}{l}\text { laparoto- } \\
\text { mia }\end{array}$ & $\begin{array}{l}\text { laparosco- } \\
\text { pia }\end{array}$ & $\begin{array}{l}\text { laparato- } \\
\text { mia }\end{array}$ & $\begin{array}{l}\text { laparato- } \\
\text { mia }\end{array}$ & $\begin{array}{l}\text { laparo- } \\
\text { tomia }\end{array}$ & $\begin{array}{l}\text { laparo- } \\
\text { tomia }\end{array}$ & $\begin{array}{l}\text { laparo- } \\
\text { tomia }\end{array}$ \\
\hline $\begin{array}{l}\text { Local de } \\
\text { biópsia }\end{array}$ & omento & peritônio & omento & $\begin{array}{l}\text { fígado/ } \\
\text { peritônio }\end{array}$ & peritônio & $\begin{array}{l}\text { apêndice/ } \\
\text { peritônio }\end{array}$ & peritônio & $\begin{array}{l}\text { per- } \\
\text { itônio }\end{array}$ & peritônio & intestino \\
\hline Histologia & SCGNC & SCGNC & SCGNC & SCGNC & SCGNC & SCGNC & SCGNC & SCGNC & SCGNC & SCGNC \\
\hline pBAAR & - & + & + & + & - & + & + & + & + & - \\
\hline
\end{tabular}

M: masculino/F: feminino; GASA: gradiente soro ascite de albumina; pBAAR: pesquisa de bacilo álcool- ácido resistente; cBAAR: cultura de bacilo álcool- ácido resistente; SCGNC: serosite crônica granulomatosa com necrose caseosa; NR: não realizado/ (+): presente/positivo; (-): ausente/negativo. 
Tabela 2 - Características clínicas dos pacientes

\begin{tabular}{ll}
\hline Características & Prevalência \\
\hline Dor abdominal & $80 \%$ \\
Febre & $60 \%$ \\
Perda de peso & $50 \%$ \\
Ascite & $50 \%$ \\
Diarréia & $30 \%$ \\
HIV & $0 \%$ \\
Tuberculose em outro sítio & $20 \%$ \\
Etilismo & $40 \%$ \\
\hline
\end{tabular}

\section{DISCUSSÃO}

A apresentação clínica da tuberculose peritoneal ocorre de forma subaguda, com sintomas aparecendo num período de semanas a meses, conforme observado neste estudo. Em estudo de metanálise, Sanai et al. revisaram relatos e séries de casos e descreveram os sinais e sintomas mais comuns em pacientes com tuberculose peritoneal. Ascite foi o sinal mais comum, sendo observada em $73 \%$ dos casos, seguido por dor abdominal $(64,5 \%)$, perda de peso (61\%) e febre (59\%). Achados de exame físico como abdômen tenso e hepatomegalia foram descritos em $47,7 \%$ e $28 \%$ dos casos, respectivamente. $^{5}$

Em nossos dados, encontramos a dor abdominal como sintoma mais freqüente, seguido por febre e perda de peso (Tabela 2). Ascite foi observada em $50 \%$ dos casos, prevalência menor em comparação com a literatura revisada. A dor abdominal deve-se, principalmente, a inflamação peritoneal e algumas vezes à oclusão do trânsito intestinal.

A comorbidade mais observada foi o etilismo, em $40 \%$ dos casos. Sabe-se que a hepatopatia alcoólica tem relação com tuberculose peritoneal. Mehta et al. mostraram que $62 \%$ dos casos de tuberculose peritoneal ocorreram em doentes com hepatopatia alcoólica. Nos países em desenvolvimento, esta relação foi encontrada em $13 \%$ dos casos. ${ }^{2}$ Nenhum paciente apresentava sorologia positiva para HIV. Outro estudo encontrou HIV positivo em $17 \%$ dos pacientes com tuberculose peritoneal. $^{8}$

Observa-se, portanto, que a presença de sintomas constitucionais como perda de peso e febre é comum, e queixas abdominais como dor abdominal, ascite e alterações de hábito intestinal são as que predominam. A falta de especificidade dos achados de anamnese e exame físico indicam a necessidade de conhecer a indicação e interpretação dos métodos complementares de diagnóstico, bem como suas vantagens, desvantagens e limitações.

Considerando a prevalência da ascite na apresentação clínica inicial, a análise do líquido ascítico costuma ser a primeira abordagem complementar. A celularidade varia entre valores inferiores a 100 células $/ \mathrm{mm}^{3}$ e superiores a 5000 células por $\mathrm{mm}^{3} .{ }^{9}$ Entretanto, a maior parte dos pacientes apresenta contagem de células entre 500 e 1500 células $/ \mathrm{mm}^{3}$, em geral, com predomínio linfocitário. No entanto, este predomínio não é um marcador de tuberculose peritoneal, mas sugere necessidade de investigação. A concentração de glicose no líquido ascítico não mostra sensibilidade significativa. A dosagem da lactatodesidrogenase no líquido ascítico superior a 900 $\mathrm{U} / \mathrm{L}$ apresenta sensibilidade de $77 \%{ }^{5}$

O gradiente soro-ascítico de albumina menor que $1,1 \mathrm{~g} / \mathrm{dl}$ é mais importante do que a dosagem isolada de proteínas, com sensibilidade de até $100 \%$ na ausência de hepatopatia crônica, porém baixa especificidade. ${ }^{10} \mathrm{~A}$ dosagem do marcador tumoral CA 125, que se eleva em doenças como neoplasia de ovário, também pode estar aumentada na tuberculose peritoneal, mas sua importância é pequena no contexto de investigação diagnóstica. ${ }^{11}$

A dosagem da adenosino desaminase (ADA), enzima liberada pelos linfócitos T e macrófagos durante a resposta imune celular a antígenos, já mostrou ser útil no diagnóstico de meningite e de efusões pleurais tuberculosas. Voigt et al. confirmaram a utilidade da mensuração da atividade da ADA em fluido ascítico da peritonite tuberculosa em dois estudos. Num primeiro estudo retrospectivo com 41 pacientes com peritonite tuberculosa, encontraram sensibilidade de $95 \%$ e especificidade de $98 \%$ e, 
em um segundo estudo, onde avaliaram prospectivamente 11 pacientes portadores de peritonite tuberculosa, a sensibilidade do exame foi de $100 \%$ e a especificidade de $96 \%$. Quando utilizaram um valor de corte de $33 \mathrm{U} / \mathrm{l}$ a sensibilidade e especificidade foram de $100 \%$ e $95 \%$, respectivamente. Com um valor de corte de $50 \mathrm{U} / \mathrm{l}$ a sensibilidade permaneceu elevada e houve menor número de resultados falso positivos. $^{12,13}$

Na presente série, a celularidade do líquido ascítico variou de 363 a 3080 células $/ \mathrm{mm}^{3}$, e todos apresentaram predomínio linfocitário. Em relação a dosagem da ADA, apenas em 1 caso dentre $5 \mathrm{com}$ dosagens realizadas, o valor foi inferior ao corte de $33 \mathrm{U} / \mathrm{l}$ (caso 8 ), com sensibilidade de $80 \%$ em nossa amostra. Esta baixa sensibilidade relativa pode ser explicada pelo pequeno tamanho da amostra. O GASA foi menor que 1,1 em apenas 3 casos em um total de 5 submetidos a paracentese diagnóstica (sensibilidade de 60\%). Entretanto, 3 destes 5 pacientes eram etilistas e um deles era portador de hepatopatia alcoólica, o que sabidamente pode alterar o gradiente e dificultar o diagnóstico.

Dados da literatura mostram que o diagnós- tico microbiológico de tuberculose peritoneal se faz através da pesquisa do bacilo no líquido ascítico em apenas $3 \%$ dos casos e através da cultura em $35 \%$, visto que a concentração do bacilo é menor do que o limiar de detecção destes métodos. ${ }^{5} \mathrm{Em}$ nossa casuística, houve crescimento do bacilo da tuberculose em cultura de líquido ascítico em apenas 1 paciente e não observamos pesquisa de bacilo positiva em nenhum caso.

Dessa forma, a pesquisa ou cultura de amostras de tecidos constitui padrão-ouro para o diagnóstico de tuberculose peritoneal. A abordagem cirúrgica quer por laparoscopia ou laparotomia, é, portanto, importante para investigação na suspeita de tuberculose peritoneal, pois permite a inspeção e a obtenção de amostras para análise histopatológica e microbiológica. $\mathrm{O}$ aspecto macroscópico característico, que consiste na presença de numerosos e diminutos nódulos esbranquiçados aderidos ao peritônio visceral e parietal (Figuras 1 e 2), apresenta correlação com o achado de serosite granulomatosa com necrose caseosa ao exame histopatológico com $93 \%$ de sensibilidade e $98 \%$ de especificidade. ${ }^{14}$

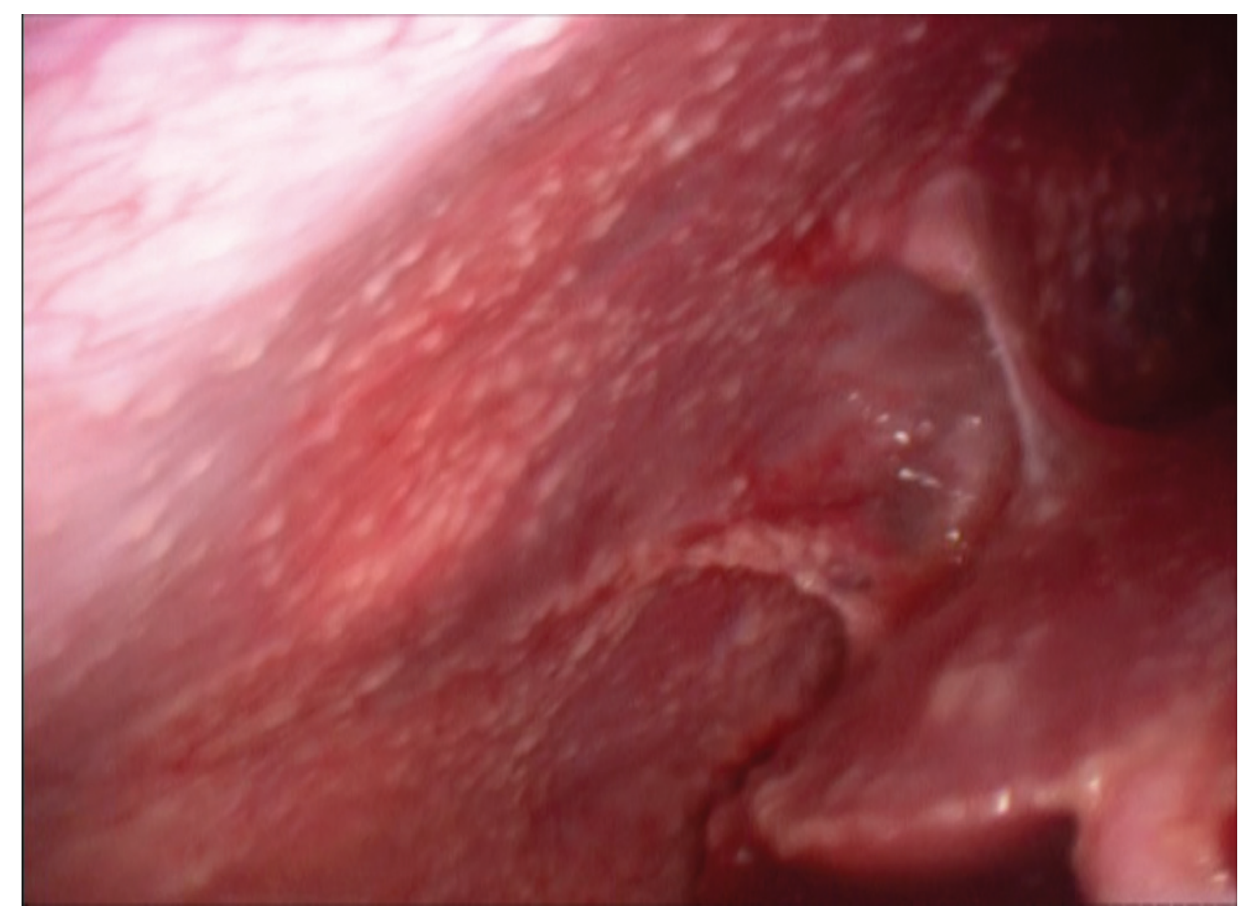

Figura 1 - Foto retirada da gravação da laparoscopia realizada no caso 2 - mostrando inúmeros nódulos de tamanhos variados de coloração esbranquiçada espalhados pelo peritônio parietal e sobre o peritônio visceral do fígado, aderências entre a cápsula hepática e o peritônio parietal. 


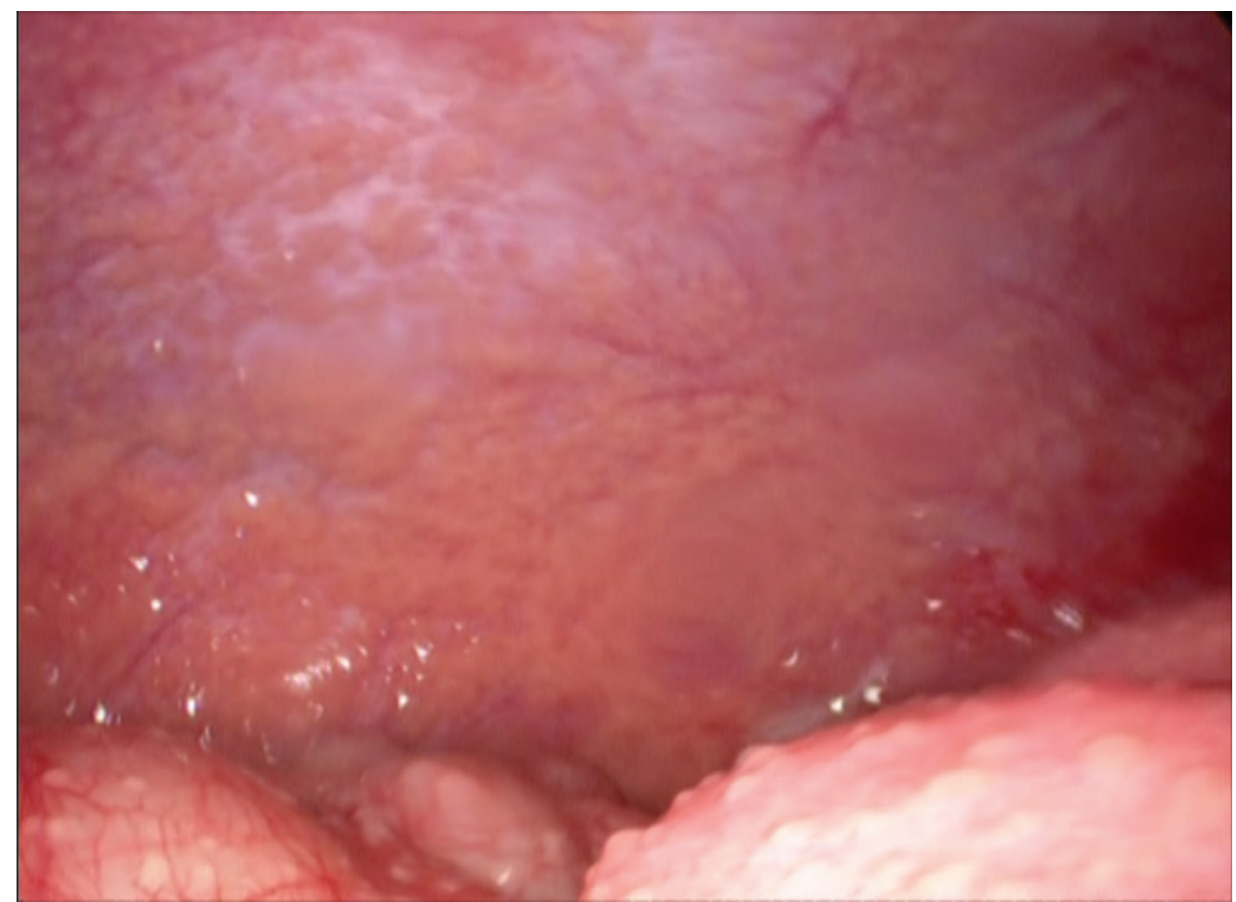

Figura 2 - Retirada da gravação da laparoscopia realizada no caso 2 - mostrando o peritônio parietal recoberto por nódulos amarelados (parte superior da figura) e nódulos sobre o epíplon que recobre as alças intestinais (parte inferior da figura).

A análise histopatológica foi determinante em todos os casos pela demonstração de serosite granulomatosa com necrose caseosa (Figura 3).
Além disso, a pesquisa do bacilo na amostra foi positiva em $70 \%$ dos casos.

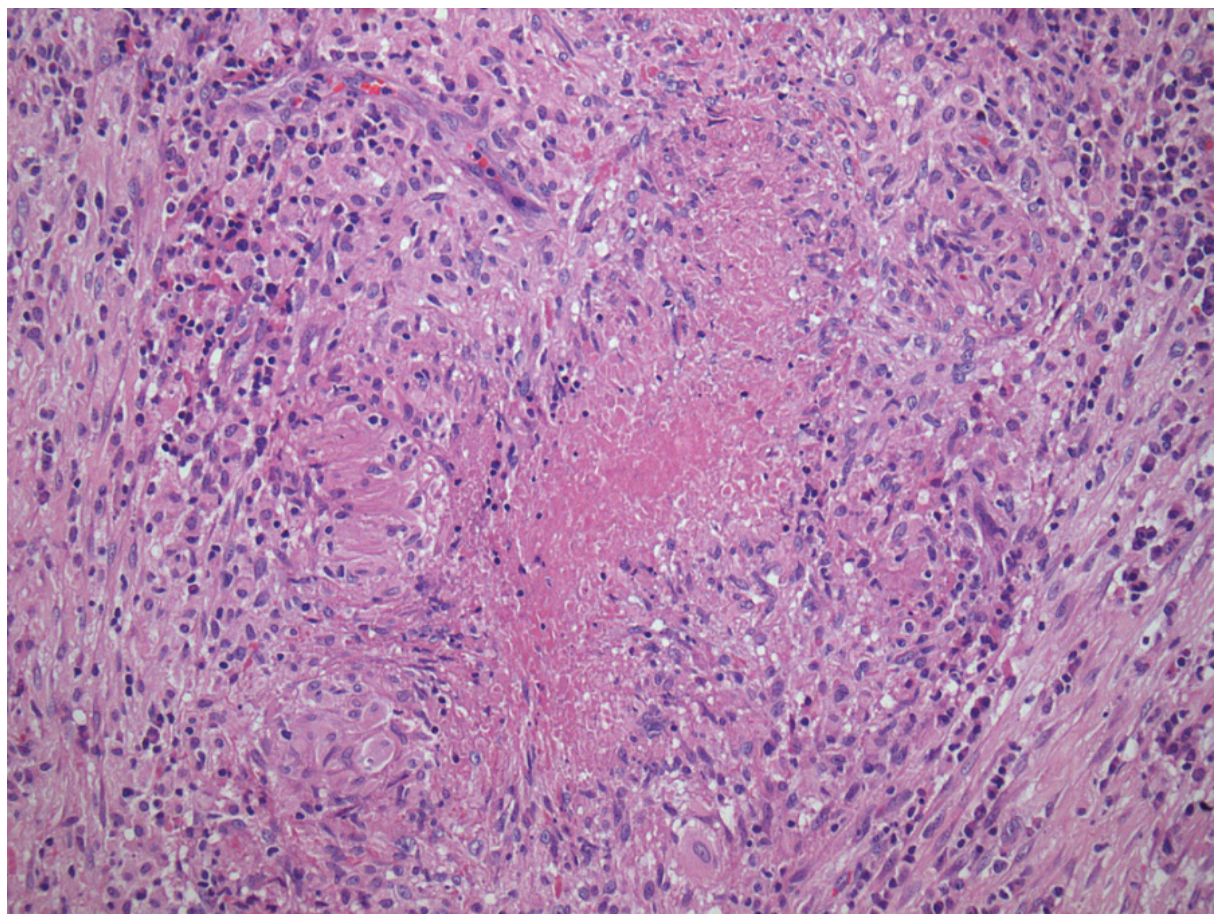

Figura 3 - Fotomicrografia - Granuloma epitelióide com necrose caseosa central (H\&E, 200x).

\section{CONCLUSÃO}

Observamos dados clínicos semelhantes à literatura. A alta prevalência de etilismo e hepatopatia alcoólica limitam a sensibilidade do gradiente sero-ascítico como ferramenta de investigação em um hospital geral. 


\section{REFERÊNCIAS}

1. Dineen P, Homan WP, Grafe WR. Tuberculous peritonitis: 43 years' experience in diagnosis and treatment. Ann Surg. 1976;184:717-22.

2. Mehta JB, Dutt A, Harvill L, Mathews KM. Epidemiology of extrapulmonary tuberculosis: a comparative analysis with pre-AIDS era. Chest. 1991;99:134-8.

3. Haas DW, DesPrez RM. Tuberculosis and acquired immunodeficiency syndrome: a historical perspective on recent developments. Am J Med. 1994;96:439-50.

4. Mimica M. The usefulness and limitations of laparoscopy in the diagnosis of tuberculous peritonitis. Endoscopy. 1992;24:588-91.

5. Sanai FM, Bzeizi KI. Systemic review: tuberculous peritonitis--Presenting features, diagnostic strategies and treatment. Aliment Pharmacol Ther. 2005;22:685-700.

6. Chu CM, Lin SM, Peng SM, Wu CS, Liaw YF. The role of laparoscopy in the evaluation of ascites of unknown origin. Gastrointest Endosc. 1994; 40:285.

7. Akpolat T. Tuberculous peritonitis. Perit Dial Int. 2009;29:166-9.

8. Tovo CV, Gama e Silva TS, Poli DD, Almeida PRL, Galperim B. Tuberculose peritoneal: análise de 12 casos. Rev
AMRIGS. 2004; 48:268-70.

9. Shakil AO, Korula J, Kanel GC, et al. Diagnostic features of tuberculous peritonitis in the absence and presence of chronic liver disease: a case control study. Am J Med. 1996;100:179-85.

10. Boyer TD. Diagnosis and management of cirrhotic ascites. In: Zakim D, Boyer TD, editors. Hepatology: a textbook of liver disease. 4th ed. Philadelphia: W.B. Saunders; 2003. p. 631-58.

11. Xiao W, Liu Y. Elevation of serum and ascites cancer antigen 125 levels in patients with liver cirrhosis. J Gastroenterol Hepatol. 2003;18:1315-6.

12. Voigt MD, Trey C, Lombard C, et al. Diagnostic value ofascites deaminase in tuberculous peritonitis. Lancet. 1989;1:751-4.

13. Hillebrand DJ, Runyon BA, Yasmineh WG, Rynders GP. Ascitic fluid adenosine deaminase insensitivity in detecting tuberculous peritonitis in the United States. Hepatology. 1996; 24:1408-12.

14. Bhargava DK, Shriniwas, Chopra $P$, et al. Peritoneal tuberculosis: laparoscopic patterns and its diagnostic accuracy. Am J Gastroenterol. 1992;87:109-12.

Conflito de Interesse: Não.

Submetido em: 12 de Maio de 2011

Aceito em: 30 de Maio de 2011

Correspondência: Divisão de Clínica Médica

Av. Prof. Lineu Prestes, 2565 - Cidade Universitária - São Paulo, SP - Brasil

CEP 05508-000 - Tel.: 55.011.3091-9200

E-mail: ffcampos@usp.br 